\title{
Delays in updates to ethics guidelines for research spark concern
}

Nearly two years ago, the US government office that oversees human research ethics launched the first-ever major revision to the so-called Common Rule, the 22-year-old regulation that governs the protection of human research subjects there. But the process set into motion by that agency-the Office of Human Research Protections (OHRP), a division of the US Department of Health and Human Services (HHS) - is dragging on. And a vocal contingent of bioethicists and researchers say the changes on the table are not enough to fix an outdated and overburdened system, advocating instead for a more fundamental rethink.

Earlier this year, many were speculating that efforts to overhaul the regulations had withered. Although the process seems to be revived, its timeframe remains uncertain. "At this point I am cautiously optimistic that we will see [the next official stage of the process] before the end of 2013, but at best I am practicing Kremlinology: guessing based on the scraps of information that escape a closed process," says Zachary Schrag, a historian at George Mason University in Washington, DC, who writes a blog about the institutional review process.

The current framework dates back to the 1970s, when Congress convened a national commission in the wake of public outcry over the Tuskegee Syphilis Study, in which federal researchers failed to inform some 400 African Americans they were afflicted with the disease or to treat them with penicillin even though the antibiotic was a known cure. On the basis of the commission's recommendations, the government issued a set of rules in 1981, including the requirement for ethical review boards and informed consent, which applied to all HHS-funded research going forward, including studies supported by the National Institutes of Health.

The framework was extended in 1991 to other federal agencies, including the Department of Veterans Affairs and the National Science Foundation, and thus became known as the 'Common Rule. However, apart from some small tweaks, the policy has remained essentially unchanged in 30 years. "The regulations have largely become calcified," Schrag says.

One sign of the drawn-out process is that the document put forth by the OHRP in July 2011 to kick off the revision process was what's known as an 'Advance Notice of Proposed Rulemaking' (ANPRM) - a sort of preproposal document-rather than a full-fledged proposal for changes to the current regulations. It posed dozens of questions to the community on issues including data protection and whether or not

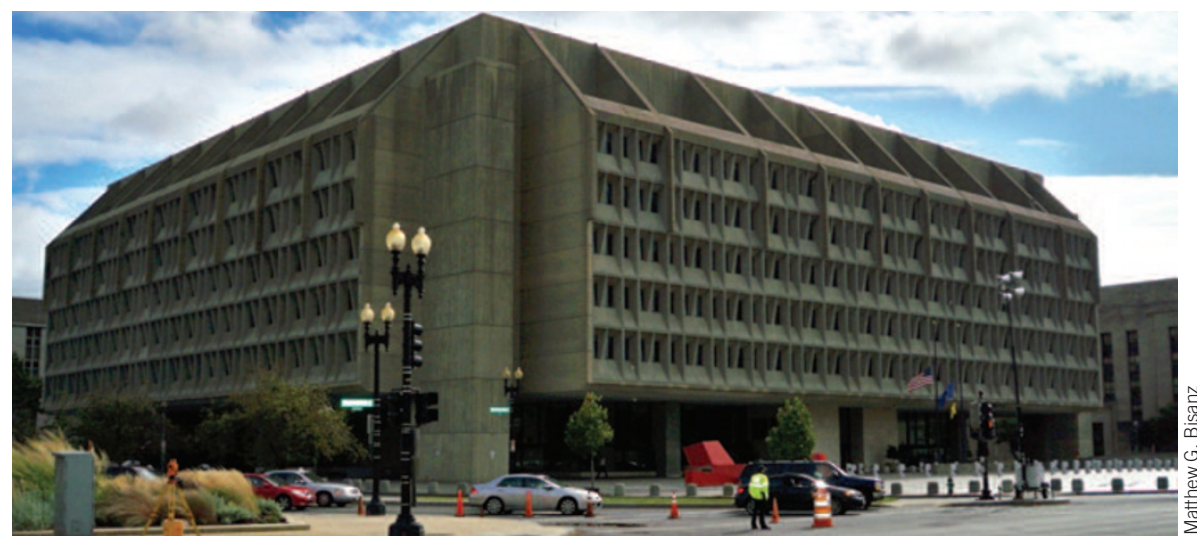

Rewriting the rules: Revisions to the Common Rule are going slowly at HHS headquarters.

the rule should be extended to apply more broadly. The document "was extraordinarily complicated and, as written, was also difficult to understand and interpret," says Barbara Bierer, senior vice president of research at Brigham and Women's Hospital in Boston, who chaired HHS's advisory committee on human research subject protections from 2008 through 2012.

The ANPRM seeks a way to address overly bureaucratic burdens that the system has accrued over time. For example, the document suggests that multisite trials-much more common today than a few decades ago-be reviewed by a single ethics board rather than by ethics boards at each institution at which part of the trial will take place (see Nat. Med. 17, 1025, 2011). The 1,142 comments accrued during the threemonth public comment period will presumably serve as the basis for the actual proposed rules. As Nature Medicine went to press, OHRP was slated to issue the proposed rules before the end of April, but the research community was not holding its breath.

\section{The definition of dispute}

On 21-22 March, the National Academy of Sciences held a workshop that took the temperature of academic scientists in regard to the proposed Common Rule revisions. Some suggested that the new regulations could provide a clearer definition of what constitutes "minimal risk" to participants in a clinical trial and provide better guidance on international trials.

But some stakeholders say that the very root of the rule needs an overhaul. Currently, any activity designed to develop or contribute to "generalizable knowledge" counts as research and requires ethics review. But that is not always a clear guide: "You can get five relatively expert people in a room and ask them what exactly constitutes research under this definition and get five different answers," says Schrag.
According to experts, the "generalizable knowledge" definition hinges on a distinction between research and clinical practice that is murky. One case in point is a hand-washing checklist implemented by Michigan hospitals a decade ago to prevent infections in intensive care units. The intervention proved so effectivesome of the poorest performing facilities cut their infection rates to zero-that the hospitals published the outcome in the New England Journal of Medicine (355, 2725-2732, 2006). But an anonymous complainant said that because the work was published, it contributed to "generalizable knowledge" and therefore constituted research-meaning that it also required informed consent from all patients involved, which had not been obtained. The OHRP agreed, spurring a storm of objections from the hospitals and others who insisted that it wasn't research in this sense but rather part of a systematic effort to improve clinical care. The OHRP ultimately backed down, but the incident cast a pall on such work.

Thirty years ago, when research primarily involved single-investigator studies of diseases and interventions, the distinction between research and practice made sense, says Robert Levine, a scholar of research ethics at Yale University in New Haven, Connecticut, who advised the 1974 commission on the topic. "That is not suitable for what's going on now," he says, because it doesn't apply to other types of disciplines such as social science and public health.

According to Jerry Menikoff, the OHRP's current director, these issues can be taken care of by the changes that will come out of the ANPRM. "Relevant agencies within HHS are currently very much engaged in working out the details of moving forward with that proposal," he told Nature Medicine in a statement.

Alla Katsnelson 